\title{
БИОАКУСТИКА
}

\author{
Н.Г. Бибиков ${ }^{1}$, С.В. Низамов ${ }^{1}$, И.Н. Пигарев ${ }^{2}$ \\ НЕЙРОННЫЕ РЕАКЦИИ КОРЫ МОЗГА КОШКИ НА ЗВУКИ, \\ ПОСТУПАЮЩИЕ С ФРОНТАЛЬНОГО НАПРАВЛЕНИЯ \\ (МЕТОДИЧЕСКИЕ АСПЕКТЫ)
}

${ }^{1}$ АО Акустический институт им. акад. Н.Н. Андреева Россия, 117036 Москва, ул. Шверника, 4, Тел.8(916)3923263,E-mail: nbibikov1@akin.ru

${ }^{2}$ Институт проблем передачи информации им. А.А. Харкевича РАН Россия, Москва, Большой Каретный, 19, E-mail: pigavev@iitp.ru.

В исследовании регистрировали нейронную активность слуховой коры кошки без применения наркотизирующих или седативных средств. Сигналы излучали высокочастотным громкоговорителем, расположенным непосредственно перед головой животного. Реакции одиночных элементов выделяли по форме импульсов в программе Spike2. Использовали специализированные программы для анализа фоновой и вызванной активности клеток.

Ключевые слова: слуховая кора, нейроны, локальные вызванные ответы, акустическая стимуляция

Роль изучения сенсорных систем для понимания работы мозга крайне существенна. Совершенно очевидно, что корковые сенсорные зоны являются важнейшим центром анализа сенсорной информации. На протяжении многих лет излюбленным объектом изучения сенсорных корковых зон являлась именно первичная слуховая кора кошек $[1,2]$. Этому отчасти способствовало ее расположение непосредственно на поверхности коры между бороздами и наличие довольно четкой тонотопической организации.

Однако подавляющее число этих работ было выполнено в условиях кетаминового, уретанового или барбиталового наркоза. Очевидно, что такие условия приводят к грубому искажению активности нейро- 
нов слуховой коры. Современные данные, полученные на грызунах и приматах $[3,4]$, позволили по-новому взглянуть на особенности ответов первичной слуховой коры, включая выраженную пластичность и способность к радикальным изменениям ответов под влиянием внимания и обучения. Между тем работы на коре интактных кошек остаются крайне немногочисленными и в нынешнем столетии выполнялись фактически в одной лаборатории $[5,6]$.

Нами была поставлена задача изучения ответов корковых слуховых зон кошки в условиях, максимально приближенных к естественным. Был использован метод безболезненной фиксации головы кошки, длительное время применявшийся нами для регистрации активности нейронов различных зон коры [например, 7]. При переходе на исследование височных зон слуховой коры эта установка, которая уже показала свою эффективность, принципиальных изменений не претерпела. Здесь мы остановимся, главным образом, на особенностях, связанных с акустической стимуляцией животного, а также рассмотрим вопрос о классификации регистрируемой активности с целью выделения отдельных нейронных элементов.

Специального рассмотрения требуют те ограничения в интерпретации наших данных, которые были обусловлены используемыми методическими приемами. Эксперименты велись в тихой, но не звукозаглушенной комнате при измерении давления вблизи ушной раковины, но не вблизи барабанной перепонки. Первое из этих ограничений, несомненно, может обусловить существенное повышение абсолютных порогов, приводя к снижению возможности количественного анализа получаемых результатов. Однако в реальной жизни животное всегда находится в активном звуковом окружении. Таким образом, наши экспериментальные условия были ближе к естественным условиям функционирования слуховой системы по сравнению с теми, которые обычно использовались в электрофизиологических экспериментах.

Что касается калибровки, то отметим, что, хотя полученные значения отличаются от измерений у барабанной перепонки, эти отличия меньше, чем в случаях использовании наркоза и полного расслабления ушных мышц. Те редкие эксперименты, в которых ушная раковина искусственно приводилась в естественное состояние [8], демонстрировали существенные изменения спектра, обусловленные наличием наружного уха.

Другая особенность проводимой работы состоит в том, что регистрируемая биологическая активность включала в себя спайки нескольких расположенных рядом нейронов. Их разделение по форме осущест- 
вляли в программе Spike 2. Примеры усредненной формы выделенных импульсов каждого класса (среднее и стандартные отклонения) приведены на рис. 1. В этом конкретном случае было выделено девять отличающихся импульсов. Три из них имели большую амплитуду (верхний ряд), а шесть - существенно меньшую. Видно, что импульсы 1 и 3 имеют близкую форму, и окончательное суждение относительно их принадлежности разным нейронам можно будет сделать после сопоставления их ответов на применяемые стимулы. Конечно, некоторое число спайков может быть классифицировано ошибочно. Однако, если не ставить задачу точного количественного описания свойств исследуемых нейронов, а ограничиться выявлением основных качественных характеристик их ответов на предъявляемые стимулы, такие погрешности можно классифицировать как статистический разброс.

Отметим также, что в каждом регистрируемом локусе определение характеристической частоты осуществляется не для конкретной клетки, а для данного кластера мультиклеточной активности. Поэтому, для некоторых, выделенных после опыта, нейронов несущая частота могла реально оказаться довольно далеко отстоящей от оптимальной. В ряде работ действительно описаны существенные нарушения тонотопической организации корковых нейронов кошки именно на микроуровне, то есть у клеток, расположенных в непосредственной близости друг от друга [4].

A
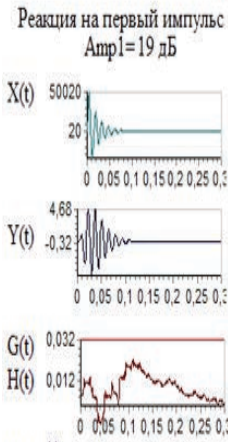

Pi(t)

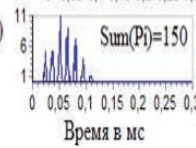

Реақция на второй импугьс Amp $2=19,5$ и $\mathrm{T}=12,8 \mathrm{Mc}$
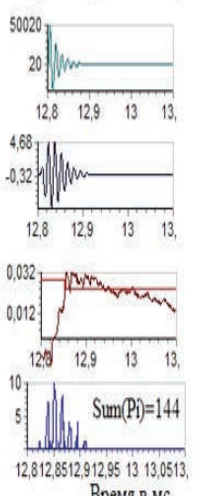

Время в мс b

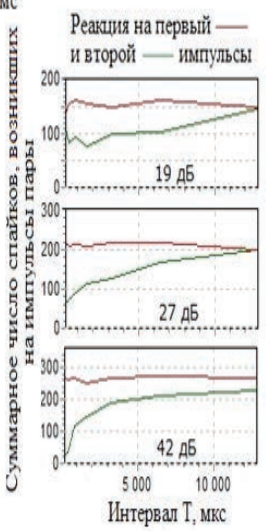

B

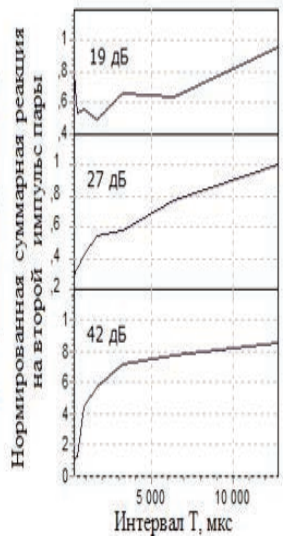

Рис. 1. Формы импульсов нейронов, выделенных программой Spike 2 в одном эксперименте. Обратите внимание, что вертикальная шкала в верхнем ряду у нейронов 1, 2 и 3 отличается от шкалы в нижних рядах. 
Мы также иногда непосредственно во время опыта отмечали нейроны, у которых оптимальная частота несущей не соответствовала оптимуму мультиклеточной активности регистрируемого локуса коры. В таких случаях мы старались исследовать реакции на сигналы с частотами, существенно отличающимися от оптимальной для данной точки регистрации, но приближающимися к характеристической частоте легко выделяемого одиночного нейрона.

Наконец, еще одно ограничение нашей работы состоит в том, что в связи со спецификой постановки длительного хронического эксперимента, не осуществляли морфологического контроля места расположения электрода. Однако, поскольку мы еще перед началом эксперимента выполняли МРТ, сканирование мозга исследуемого животного, и создавали индивидуальный стереотаксический атлас, можно было достаточно точно рассчитать траекторию перемещения микроэлектродов. Кроме того, сами особенности реакции клеток, регистрируемых в процессе движения микроэлектродов, несомненно свидетельствовали именно о прохождении через зону первичной слуховой коры с оптимальными частотами порядка 8-13 кГц.

Мы обнаружили некоторые особенности полученных нами данных по сравнению с теми результатами, которые были известны ранее относительно свойств нейронов в слуховой коре кошек. Основное из них состояло том, что в наших экспериментах часто наблюдались устойчивые ответы нейронов на весьма длительные тональные сигналы, модулированные низкочастотным шумом. Стоит подчеркнуть, что именно такая и только такая информация поступает в слуховой анализатор непосредственно после преобразования текущего входного сигнала во внутреннем ухе. Это определяется тем очевидным фактом, что сигнал на выходе узкополосного фильтра, расположенного на улитке, можно рассматривать как синусоиду, соответствующую его частоте настройки, модулированную низкочастотным фильтром в полосе от нуля до частоты, соответствующей ширине фильтра. Между тем в большинстве предшествующих работ многократно подчеркивалась весьма слабая способность нейронов коры следовать за изменениями амплитуды сигнала $[1,2]$. Даже в работах, выполненных без анестезии, обычно исследовали ответы на сравнительно редко предъявляемые или очень медленно меняющиеся стимулы $[5,6]$.

Исследованные в работе клетки, как правило, весьма эффективно реагировали не только на амплитудно-модулированные тональные сигналы, но и на сложные звуковые стимулы, предположительно связанные с жизнедеятельностью животного. В число этих сигналов входили звуки, 
XVII Школа-семинар им. акад. Л.М. Бреховских «Акустика океана», XXXIII сессия РАО

издаваемые мелкими грызунами, а также певчими птицами. При этом можно было оценить специфичность этих реакций, которая у некоторых клеток была весьма высока.

Работа была частично поддержана грантом РФФИ № 19-04-00215.

\title{
Литература
}

1 Kim S.-J., Manyam S.C., Warren D.J., Normann R.A. Electrophysiological mapping of cat primary auditory cortex with multielectrode arrays // Annals of Biomedical Engineering. 2006. Vol. 34. P. 300-309.

2. Gourévitch B., Noreña A., Shaw G., Eggermont J.J. Spectrotemporal receptive fields in anesthetized cat primary auditory cortex are context dependent // Cerebral Cortex. 2009. Vol. 19, P. 1448-1461. DOI: 10.1093/cercor/bhn184.

3. Downer J.D., Niwa M., Sutter M.L. Task engagement selectively modulates neural correlations in primary auditory cortex // Journal of Neuroscience. 2015. Vol. 35. P. 7565-7574. DOI: 10.1523/jneurosci.4094-4114.

4. Rothschild G., Nelken I., Mizrahi, A. Functional organization and population dynamics in the mouse primary auditory cortex // Nature Neuroscience. 2010. Vol. 13. P. 353-360.

5. Qin L., Kitama T., Chimoto S., Sakayori S., Sato Y. Time course of tonal frequency-responsearea of primary auditory cortex neurons in alert cats // Neuroscience Research. 2003. Vol. 46. P. 145-152. DOI: 10.1016/s0168-0102(03)00034-8.

6. Dong C., Qin L., Liu Y., Zhang X., Sato Y. Neural responses in the primary auditory cortex of freely behaving cats while discriminating fast and slow click-trains // PLoS ONE. 2011. Vol. 6: e25895. DOI: 10.1371/journal.pone.0025895.

7. Бибиков Н.Г., Пигарев И.Н.Фоновая активность одиночных нейронов коры спящей кошки // Физиологический журнал 2013. Вып. 3. С. 348-362.

8. Imig T.J., Bibikov N.G., Pourrier P., Samson F.K. Directionality derived from pinnacue spectral notches in cat dorsal cochlear nucleus // J. Neurophysiol. 2000. Vol. 83. P. 907-925.

N.G. Bibikov ${ }^{1}$, S.V. Nizamov ${ }^{1}$, I.N. Pigarev $^{2}$

\section{INVESTIGATION OF THE NEURONAL RESPONSES TO FRONTAL SOUNDS IN TEMPORAL AUDITORY CORTEX OF CATS (METHODICAL ASPECTS)}

\author{
${ }^{1}$ JSE N.N. Andreev Acoustical Institute \\ Russia, 117036 Moscow, st. Shvernik, 4, \\ Tel.8(916)3923263E-mail: nbibikov1@yandex.ru \\ ${ }^{2}$ Institute for Information Transmission Problems (Kharkevich Institute) \\ RAS.E-mail: pigarev@iitp.ru
}

The neuronal activity in the auditory cortex of a cat was recorded without the use of anesthetics or sedatives. The sounds were emitted by a high frequency loudspeaker located in front of the animal's head. The reactions of single elements were classified accordingly to the spike shape by the Spike2 
program. We used specialized programs for the analysis of background and evoked cell activity.

Keywords: auditory cortex, neurons, local field potentials, acoustic stimulation, spike sorting

Acknowledgment: This work was partially supported by the RFBR grant No. 19-04-00215.

\section{References}

1. Kim S.-J., Manyam S.C., Warren D.J., and Normann R.A. Electrophysiological mapping of cat primary auditory cortex with multielectrode arrays. Annals of Biomedical Engineering, 2006, Vol. 34, pp. 300-309.

2. Gourévitch B., Noreña A., Shaw G., and Eggermont J.J. Spectrotemporal receptive fields in anesthetized cat primary auditory cortex are context dependent. Cerebral Cortex, 2009, Vol. 19, pp. 1448-1461, doi: 10.1093/cercor/bhn184.

3. Downer J.D., Niwa M., \& Sutter M.L. Task engagement selectively modulates neural correlations in primary auditory cortex. Journal of Neuroscience, 2015, Vol. 35, pp. 75657574, doi: 10.1523/jneurosci.4094-4114.

4. Rothschild G., Nelken I., and Mizrahi A. Functional organization and population dynamics in the mouse primary auditory cortex. Nature Neuroscience, 2010, Vol. 13, pp. 353-360.

5. Qin L., Kitama T., Chimoto S., Sakayori S., \& Sato Y. Time course of tonal frequencyresponse-area of primary auditory cortex neurons in alert cats. Neuroscience Research, 2003, Vol. 46(2), pp. 145-152, doi: 10.1016/s0168-0102(03)00034-8.

6. Dong C., Qin L., Liu Y., Zhang X., and Sato Y. Neural responses in the primary auditory cortex of freely behaving cats while discriminating fast and slow click-trains. PLoS ONE, 2011, Vol. 6, e25895, doi: 10.1371/journal.pone.0025895.

7. Bibikov N.G. and Pigarev I.N. The statistical properties of the background activity of cortical neurons in cats during slowwave sleep. Neuroscience and Behavioral Physiology, 2015, Vol. 45, No. 2, pp. 213-222.

8. Imig T.J., Bibikov N.G., Pourrier P., Samson F.K. Directionality derived from pinna-cue spectral notches in cat dorsal cochlear nucleus. J. Neurophysiol., 2000, Vol. 83, pp. 907-925. 